\title{
On the minimal degree of a transitive permutation group with stabilizer a 2-group
}

\author{
Primož Potočnik and Pablo Spiga* \\ Communicated by Andrea Lucchini
}

\begin{abstract}
The minimal degree of a permutation group $G$ is defined as the minimal number of non-fixed points of a non-trivial element of $G$. In this paper, we show that if $G$ is a transitive permutation group of degree $n$ having no non-trivial normal 2-subgroups such that the stabilizer of a point is a 2-group, then the minimal degree of $G$ is at least $\frac{2}{3} n$. The proof depends on the classification of finite simple groups.
\end{abstract}

\section{Introduction}

Given a finite group acting on a finite set $\Omega$ and $g \in G$, we let

$$
\operatorname{Fix}_{\Omega}(g):=\left\{\omega \in \Omega \mid \omega^{g}=\omega\right\}
$$

denote the set of fixed points of $g$, and we let $\operatorname{supp}_{\Omega}(g):=\Omega \backslash \operatorname{Fix}_{\Omega}(g)$. Then the parameter

$$
\operatorname{mindeg}_{\Omega}(G):=\min _{g \in G \backslash\{1\}}\left|\operatorname{supp}_{\Omega}(g)\right|
$$

is called the minimal degree of the permutation group $G$. Minimal degree (and a related parameter $\operatorname{Fix}_{\Omega}(G):=\max _{g \in G \backslash\{1\}}\left|\operatorname{Fix}_{\Omega}(g)\right|=|\Omega|-\operatorname{mindeg}_{\Omega}(G)$, sometimes called the fixity of $G$ ) has been the focus of intense study by several authors with most work concentrating on proving upper and lower bounds on the minimal degree of primitive permutation groups and permutation actions of classical groups (see $[2,5,13,15,20]$, to name a few). Far fewer papers deal with other permutation groups (see $[18,23])$.

The aim of this paper is to consider a lower bound on the minimal degree of a transitive permutation group $G$ whose point stabilizer is a 2-group and such that $\mathbf{O}_{2}(G)=1$ (where by $\mathbf{O}_{2}(G)$ we denote the largest normal 2-subgroup of $G$ ). In particular, we prove the following.

The first-named author gratefully acknowledges the support of the Slovenian Research Agency ARRS, core funding programme P1-0294 and research project J1-1691. 
Theorem 1.1. Let $G$ be a transitive permutation group on a set $\Omega$ with $\mathbf{O}_{2}(G)=1$ such that the point stabilizer $G_{\omega}$ is a 2-group. Then $\operatorname{mindeg}_{\Omega}(G) \geq \frac{2}{3}|\Omega|$.

Our motivation to prove this result stems from the investigation of the minimal degree of permutation groups appearing as arc-transitive automorphism groups of finite connected (possibly directed) graphs or, equivalently, permutation groups admitting a connected suborbit, where by a connected suborbit of a transitive permutation group $G \leq \operatorname{Sym}(\Omega)$ we mean an orbit $\Sigma$ of the point stabilizer $G_{\omega}$ acting on $\Omega \backslash\{\omega\}$, such that the directed graph with vertex-set $\Omega$ and edge-set $\left\{\left(\omega^{g}, \delta^{g}\right) \mid \delta \in \Sigma, g \in G\right\}$ is connected. Note that a transitive permutation group $G \leq \operatorname{Sym}(\Omega)$ is primitive if and only if all of its non-trivial suborbits are connected. In view of the numerous results regarding the minimal degree of permutation groups, it is thus natural to relax the condition of primitivity to that of an existence of a single connected suborbit. In fact, based on the available computational data $[10,11,25,26]$, one can see that, under some additional assumptions on the length and the self-pairedness of the connected suborbit (and modulo some well-defined exceptions), lower bounds on the minimal degree holding for primitive permutation groups seem to extend to this more general situation. Theorem 1.1 represents a crucial step in a recent result [24] stating that, apart from a wellunderstood infinite family and a finite set of examples, every transitive permutation group $G \leq \operatorname{Sym}(\Omega)$ admitting a connected suborbit of length at most 2 or a selfpaired connected suborbit of length at most 4 satisfies $\operatorname{mindeg}_{\Omega}(G) \geq \frac{2}{3}|\Omega|$ (a suborbit $\Sigma$ is self-paired if, for every edge $\left(\omega^{\prime}, \omega^{\prime \prime}\right)$ of the corresponding directed graph, also the pair $\left(\omega^{\prime \prime}, \omega^{\prime}\right)$ is an edge of that directed graph). Arc-transitive automorphism groups of graphs having point stabilizer a 2-group have already cropped up a few times in the literature; see for instance [31,32].

We would also like to point out that Guralnick and Magaard [16] (building on earlier work of Lawther, Liebeck and Saxl [20,21]) have proved that, except for an explicit list of exceptions, the minimal degree of every primitive permutation group of degree $n$ is at least $\frac{n}{2}$. Our proof of Theorem 1.1 quickly reduces to the case that the group $G$ under consideration is quasiprimitive. In this sense, our result can be thought of as an attempt to extend the result of Guralnick and Magaard to the case of quasiprimitive groups.

We use fairly standard notation. Given a set $\Omega$, we denote by $\operatorname{Sym}(\Omega)$ and $\operatorname{Alt}(\Omega)$ the symmetric and the alternating group on $\Omega$. When the domain $\Omega$ is irrelevant or clear from the context, we write $\operatorname{Sym}(n)$ and $\operatorname{Alt}(n)$ for the symmetric and alternating group of degree $n$. Given a permutation $g \in \operatorname{Sym}(\Omega)$, we write $\operatorname{fpr}_{\Omega}(g)$ for the fixed-point ratio of $g$, that is,

$$
\operatorname{fpr}_{\Omega}(g):=\frac{\left|\operatorname{Fix}_{\Omega}(g)\right|}{|\Omega|} .
$$


Using this notation, Theorem 1.1 is equivalent to the following.

Theorem 1.2. Let $G$ be a transitive permutation group on a set $\Omega$ with $\mathbf{O}_{2}(G)=1$ such that the point stabilizer $G_{\omega}$ is a 2-group. Then $\operatorname{fpr}_{\Omega}(g) \leq \frac{1}{3}$ for every nonidentity element $g$ of $G$.

Sometimes, for instance in Lemma 2.1, we need slightly more general notation: given a subset $\Delta$ of $\Omega$, we let $\operatorname{fpr}_{\Delta}(g):=\frac{\left|\Delta \cap \operatorname{Fix}_{\Omega}(g)\right|}{|\Delta|}$.

A subgroup $G$ of $\operatorname{Sym}(\Omega)$ is said to be semiregular if the identity is the only element of $G$ fixing some point of $\Omega$. Let $G$ be a group, and let $H$ be a subgroup of $G$; we denote by $H \backslash G$ the set of right cosets of $H$ in $G$. Recall that $G$ acts transitively on $H \backslash G$ by right multiplication. The normalizer of $H$ in $G$ is denoted by $\mathbf{N}_{G}(H)$.

\section{Proof of Theorem 1.1}

We begin with two useful lemmas and then proceed to the proof of Theorem 1.1.

Lemma 2.1. Let $X$ be a group acting transitively on $\Omega$, let $\Sigma$ be a system of imprimitivity for $X$ in its action on $\Omega$, and let $x \in X$. Then $\operatorname{fpr}_{\Omega}(x) \leq \operatorname{fr}_{\Sigma}(x)$.

Proof. This is clear because, if $x$ fixes a block $B \in \Sigma$, then $\operatorname{Fix}_{B}(x) \subseteq B$ and hence $\left|\operatorname{Fix}_{B}(x)\right| \leq|B|$.

Lemma 2.2. Let $X$ be a group acting on a set $\Omega$, let $Y$ be a normal subgroup of $X$, let $x \in X$, and let $\omega \in \Omega$. Then

$$
\operatorname{fpr}_{\omega^{Y}}(x)=\frac{\left|x^{Y} \cap X_{\omega}\right|}{\left|x^{Y}\right|},
$$

where $x^{Y}:=\left\{x^{y} \mid y \in Y\right\}$ is the $Y$-conjugacy class of the element $x$.

Proof. This equality is classic; see for instance [21]. Here we present a short proof: consider the bipartite graph with one side of the bipartition labeled by the elements of $x^{Y}$ and the other side of the bipartition labeled by the elements of $\omega^{Y}$. Declare $x^{\prime} \in x^{Y}$ adjacent to $\omega^{\prime}$ if $x^{\prime}$ fixes $\omega^{\prime}$. Clearly, $x^{\prime} \in x^{Y}$ has valency $\left|\operatorname{Fix}_{\omega^{Y}}\left(x^{\prime}\right)\right|=\left|\operatorname{Fix}_{\omega^{Y}}(x)\right|$ and $\omega^{\prime} \in \omega^{Y}$ has valency $\left|x^{Y} \cap X_{\omega^{\prime}}\right|=\left|x^{Y} \cap X_{\omega}\right|$. Thus $\left|x^{Y}\right|\left|\operatorname{Fix}_{\omega^{Y}}(x)\right|=\left|x^{Y} \cap X_{\omega} \| \omega^{Y}\right|$.

The rest of the section is devoted to the proof Theorem 1.1. Let $G$ be a transitive permutation group acting on a set $\Omega,|\Omega|=n$, with $\mathbf{O}_{2}(G)=1$ such that the point 
stabilizer $G_{\omega}$ is a 2-group. We need to prove that $\operatorname{fix}_{\Omega}(G) \leq \frac{n}{3}$. Our proof is by induction on $|\Omega|+|G|$.

Let $Q$ be a Sylow 2-subgroup of $G$ with $G_{\omega} \leq Q$, and let $B:=\omega^{Q}$. Since $G_{\omega} \leq Q$, the set $\Sigma:=\left\{B^{x} \mid x \in G\right\}$ is a $G$-invariant partition of $\Sigma$ upon which $G$ acts with the stabilizer of the element $B \in \Sigma$ being $Q$. As $\mathbf{O}_{2}(G)=1$ and $Q$ is a 2-group, the action of $G$ on $\Sigma$ is faithful. If $|\Sigma|<|\Omega|$, then by induction $\operatorname{fpr}_{\Sigma}(g) \leq \frac{1}{3}$ for every $g \in G \backslash\{1\}$. Therefore, from Lemma 2.1, we have $\operatorname{fpr}_{\Omega}(g) \leq \frac{1}{3}$ for every $g \in G \backslash\{1\}$. We may thus suppose that $Q=G_{\omega}$, that is,

$$
G_{\omega} \text { is a Sylow 2-subgroup of } G \text {. }
$$

Let $N$ be a minimal normal subgroup of $G$, and let $K$ be the kernel of the action of $G$ on the set $\Sigma$ of $N$-orbits. Suppose that $N$ is not transitive on $\Omega$. In particular, $G / K$ is a non-identity transitive permutation group on $\Sigma$ of odd degree greater than 1 ; moreover, given $B \in \Sigma$ and $\omega \in B$, the setwise stabilizer of $B$ in $G$ is $G_{\omega} K$, and $G_{\omega} K / K$ is a Sylow 2-subgroup of $G / K$. By induction, if $g \in G \backslash K$, then $\operatorname{fpr}_{\Sigma}(g K) \leq \frac{1}{3}$, and hence $\operatorname{fpr}_{\Omega}(g) \leq \operatorname{fpr}_{\Sigma}(g K) \leq \frac{1}{3}$. Suppose that $g \in K \backslash\{1\}$. Since $K \unlhd G$ and $G_{\omega}$ is a Sylow 2-subgroup of $G$, we deduce that $K_{\omega}$ is a Sylow 2-subgroup of $K$. In particular, from the Frattini argument, we have $G=K \mathbf{N}_{G}\left(K_{\omega}\right)$. This implies that the core of $K_{\omega}$ in $K$ equals the core of $K_{\omega}$ in $G$, which is trivial, implying that $K$ acts faithfully on $\omega^{K}$. Since this argument does not depend upon $\omega \in \Omega, K$ acts faithfully on each element of $\Sigma$. In particular, for every $B \in \Sigma$, the restriction of $g$ to $B$ is a non-identity permutation, and hence, by induction, $\operatorname{fp}_{B}(g) \leq \frac{1}{3}$. Since this argument does not depend upon $B \in \Sigma$, we have $\operatorname{fpr}_{\Omega}(g) \leq \frac{1}{3}$.

It remains to deal with the case that every minimal normal subgroup of $G$ is transitive on $\Omega$, that is, $G$ is quasiprimitive.

The class of quasiprimitive permutation groups may be described (see [27]) in a fashion very similar to the description given by the O'Nan-Scott theorem for primitive permutation groups. In [28], this description is refined, and eight types of quasiprimitive groups are defined, namely HA, HS, HC, SD, CD, TW, PA and AS, such that every quasiprimitive group belongs to exactly one of these types. As our group $G$ has odd degree, it is readily seen, using the terminology in $[27,28]$, that it is of HA, AS or PA type, that is, holomorphic abelian, almost simple or product action. We refer the reader to $[27,28]$ for more information on the structure of groups of HA, AS or PA type, or to [29] for an extensive treatment of permutation groups and Cartesian decompositions.

Assume $G$ has O'Nan-Scott type HA; let $V$ be the socle of $G$, let $g \in G \backslash\{1\}$ with $\operatorname{Fix}_{\Omega}(g) \neq \emptyset$, and let $\omega \in \operatorname{Fix}_{\Omega}(g)$. As $\mathbf{O}_{2}(G)=1, V$ is an elementary abelian $p$-group for some prime $p>2$. Then $G=V \rtimes G_{\omega}$, and the action of $G$ on $\Omega$ 
is permutation equivalent to the natural holomorph action of $G$ on $V$, with $V$ acting by right multiplication and with $G_{\omega}$ acting by conjugation. Using this identification, we have

$$
\operatorname{fpr}_{\Omega}(g)=\frac{1}{\left|V: \mathbf{C}_{V}(g)\right|} \leq \frac{1}{p} \leq \frac{1}{3} .
$$

Assume that $G$ has O'Nan-Scott type PA. Following [27,28], we set some notation and state some facts regarding the groups of O'Nan-Scott type PA. Let $g \in G \backslash\{1\}$ with $\operatorname{Fix}_{\Omega}(g) \neq \emptyset$, let $\omega \in \operatorname{Fix}_{\Omega}(g)$, and let $N$ be the socle of $G$. There exists a finite non-abelian simple group $T$ such that $N=T_{1} \times T_{2} \times \cdots \times T_{\ell}$ for some $\ell \geq 2$ with $T_{i} \cong T$ for each $i$. The group $G$ can then be identified with a subgroup of $\operatorname{Aut}(T)$ wr $\operatorname{Sym}(\ell)$. Let $R$ be a Sylow 2-subgroup of $T$, and let $\Delta$ be the set $R \backslash T$ of right cosets of $R$ in $T$. As $G_{\omega}$ is a Sylow 2-subgroup of $G$, we have $G_{\omega} \cap N=R_{1} \times \cdots \times R_{\ell} \cong R^{\ell}$, where $R_{i}$ is a Sylow 2-subgroup of $T_{i}$ for each $i$. From [27,28], the action of $G$ on $\Omega$ is permutation isomorphic to the natural Cartesian product action of $G$ on $\Delta^{\ell}$. By identifying $\Omega$ with $\Delta^{\ell}$, we have $G \leq W$ with $W:=\operatorname{Aut}(T)$ wr $\operatorname{Sym}(\ell)$, where $W$ acts on $\Omega$ with the Cartesian product action. In particular, we may write the elements $x \in W$ in the form $x=\left(a_{1}, a_{2}, \ldots, a_{\ell}\right) \sigma$ for some $a_{1}, a_{2}, \ldots, a_{\ell} \in \operatorname{Aut}(T)$ and $\sigma \in \operatorname{Sym}(\ell)$. Recall that if $\left(\delta_{1}, \delta_{2}, \ldots, \delta_{\ell}\right) \in \Delta^{\ell}=\Omega$, then

$$
\begin{aligned}
\left(\delta_{1}, \delta_{2}, \ldots, \delta_{\ell}\right)^{x} & =\left(\delta_{1}, \delta_{2}, \ldots, \delta_{\ell}\right)^{\left(a_{1}, a_{2}, \ldots, a_{\ell}\right) \sigma}=\left(\delta_{1}^{a_{1}}, \delta_{2}^{a_{2}}, \ldots, \delta_{\ell}^{a_{\ell}}\right)^{\sigma} \\
& =\left(\delta_{1 \sigma^{-1}}^{a_{1 \sigma^{-1}}}, \delta_{2 \sigma^{-1}}^{a_{2 \sigma^{-1}}}, \ldots, \delta_{\ell \sigma^{-1}}^{a_{\ell-1}}\right) .
\end{aligned}
$$

We write $g=\left(a_{1}, a_{2}, \ldots, a_{\ell}\right) \sigma$.

Suppose $\sigma$ is a cycle of length $\kappa \geq 2$. Relabeling the index set $\{1, \ldots, \ell\}$, we may suppose that $\sigma=(12 \cdots \kappa)$. From $(2.2)$, we see that $\left(\delta_{1}, \ldots, \delta_{\ell}\right) \in \Omega$ is fixed by $g$ if and only if

$$
\delta_{2}=\delta_{1}^{a_{1}}, \delta_{3}=\delta_{2}^{a_{2}}, \ldots, \delta_{\kappa}=\delta_{\kappa-1}^{a_{\kappa-1}} \delta_{\kappa+1}^{a_{\kappa+1}}, \ldots, \delta_{\ell}=\delta_{\ell}^{a_{\ell}} .
$$

Clearly, this yields that the points fixed by $g$ are of the form

$$
\left(\delta_{1}, \delta_{1}^{a_{1}}, \delta_{1}^{a_{1} a_{2}}, \ldots, \delta_{1}^{a_{1} a_{2} \cdots a_{\kappa-1}}, \delta_{\kappa+1}, \ldots, \delta_{\ell}^{a_{\ell}}\right),
$$

where $\delta_{1}$ is an arbitrary element of $\Delta$ and $\delta_{i} \in \operatorname{Fix}_{\Delta}\left(a_{i}\right)$ for each $i>\kappa$. Therefore,

$$
\operatorname{fpr}_{\Omega}(g)=\frac{|\Delta|}{|\Delta|^{\kappa}} \cdot \prod_{j=\kappa+1}^{\ell} \operatorname{fpr}_{\Delta}\left(a_{i}\right) \leq \frac{1}{|\Delta|^{\kappa-1}} \leq \frac{1}{|\Delta|} .
$$

Suppose now $\sigma \neq 1$. Arguing by induction on the number of cycles in a decomposition of $\sigma$ in disjoint cycles and using a computation similar to that in the 
previous paragraph, we obtain

$$
\operatorname{fpr}_{\Omega}(g) \leq \frac{1}{|\Delta|}
$$

As $|\Delta|=|T: R|$ and $T$ is a non-abelian simple group, we have $|\Delta| \geq 3$ and hence $\operatorname{fpr}_{\Omega}(g) \leq 3$.

Suppose $\sigma=1$. As $g \neq 1$, without loss of generality, we may assume $a_{1} \neq 1$. Then

$$
\operatorname{Fix}_{\Omega}(g)=\operatorname{Fix}_{\Delta^{\ell}}(g) \subseteq \operatorname{Fix}_{\Delta}\left(a_{1}\right) \times \Delta^{\ell-1} .
$$

Since $\ell \geq 2$, we have $|\Delta|<|\Omega|$ and hence, by induction,

$$
\operatorname{fpr}_{\Delta}\left(a_{1}\right) \leq \frac{1}{3} \quad \text { and } \quad \operatorname{fpr}_{\Omega}(g) \leq \frac{1}{3} .
$$

It remains to deal with the case that $G$ is an almost simple group. Let $T$ be the socle of $G$. We now divide the proof in four parts, depending on whether $T$ is a sporadic, an alternating, an exceptional group of Lie type or a classical group.

\subsection{Sporadic groups}

Here we consider the situation where the socle $T$ is one of the sporadic finite simple groups. The proof is entirely computational and uses the astonishing package "The GAP character Table Library" [1] implemented in the computer algebra system GAP [33]. For sporadic groups, the proof of Theorem 1.1 follows immediately from Lemma 2.1 and from Lemma 2.3.

Lemma 2.3. Let $G$ be an almost simple primitive group on $\Omega$ with socle a sporadic simple group. Then

$$
\operatorname{fpr}_{\Omega}(g) \leq \frac{1}{3}
$$

for every $g \in G \backslash\{1\}$, except when $G=\operatorname{Aut}\left(M_{22}\right)$ in its primitive action of degree 22, where the maximum fixed-point ratio is $\frac{4}{11}$. Moreover, for each 2-subgroup $Q$ of $\operatorname{Aut}\left(M_{22}\right)$ and for each $x \in \operatorname{Aut}\left(M_{22}\right)$ with $g \neq 1$, in the action of $\operatorname{Aut}\left(M_{22}\right)$ on the set $Q \backslash \operatorname{Aut}\left(M_{22}\right)$ of cosets of $Q$ in $\operatorname{Aut}\left(M_{22}\right)$, we have

$$
\operatorname{fpr}_{Q \backslash \operatorname{Aut}\left(M_{22}\right)}(g) \leq \frac{3}{55}<\frac{1}{3} .
$$

Proof. Apart from

- the Monster and

- the action of the Baby Monster on the cosets of a maximal subgroup of type $\left(2^{2} \times F_{4}(2)\right): 2$, 
each permutation character of each primitive permutation representation of an almost simple group with socle a sporadic simple group is available in GAP via the package "The GAP character Table Library". Therefore, except for the two cases mentioned above, we can quickly and easily use GAP to test the veracity of the lemma. The permutation character of the Baby Monster $G$ on the cosets of a maximal subgroup $M$ of type $\left(2^{2} \times F_{4}(2)\right): 2$ is missing from the GAP library because the conjugacy fusion of some of the elements of $M$ in $G$ remains a mystery; this information is vital for computing the permutation character.

In the rest of the proof, we use Lemma 2.2. Suppose then $G$ is the Baby Monster. Let $G_{\omega}$ be the stabilizer in $G$ of the point $\omega \in \Omega$, and suppose

$$
G_{\omega} \cong\left(2^{2} \times F_{4}(2)\right): 2 .
$$

Using the ATLAS notation, with a direct computation, we see that

$$
\operatorname{fpr}_{\Omega}(g)=\frac{\left|g^{G} \cap G_{\omega}\right|}{\left|g^{G}\right|} \leq \frac{\left|G_{\omega}\right|}{\left|g^{G}\right|} \leq \frac{1}{3},
$$

unless $g$ is in the conjugacy class $1 A, 2 A$ or $2 B$. In particular, the lemma is proved also in this case, except when $g$ is in the conjugacy class $2 A$ or $2 B$. Let us denote by $t$ the number of solutions to the equation $x^{2}=1$ in $G_{\omega}$. From [17, (4.6)], we see that

$$
t=\sum_{\chi \in \operatorname{Irr}^{+}\left(G_{\omega}\right)} \chi(1)-\sum_{\chi \in \operatorname{Irr}^{-}\left(G_{\omega}\right)} \chi(1),
$$

where $\operatorname{Irr}^{+}\left(G_{\omega}\right)$ and $\operatorname{Irr}^{-}\left(G_{\omega}\right)$ are the sets of the irreducible complex characters of $G_{\omega}$ of orthogonal and of symplectic type. As the character table of $G_{\omega}$ is available in GAP, we can compute $t$ with this formula, and we obtain that $t=1605784576$. Therefore, when $g$ is in the conjugacy class $2 A$ and $2 B$, we can refine the previous bound, and we obtain

$$
\operatorname{fpr}_{\Omega}(g)=\frac{\left|g^{G} \cap G_{\omega}\right|}{\left|g^{G}\right|} \leq \frac{t}{\left|g^{G}\right|} \leq \frac{1}{3} .
$$

For the rest of this proof, we may assume that $G$ is the Monster group. Let $\omega \in \Omega$. From [30, Section 3.6], we see that the classification of the maximal subgroups of the Monster is complete except for a few small open cases. Suppose first that $G_{\omega}$ is isomorphic to the double cover of the Baby monster. Let $\pi$ be the permutation character of $G$ on $\Omega$. It was proved by Breuer and Lux [4, page 2309] that

$$
\pi=\chi_{1}+\chi_{2}+\chi_{4}+\chi_{5}+\chi_{9}+\chi_{14}+\chi_{21}+\chi_{34}+\chi_{35}
$$


(this was also proved independently in [22]). With this character, we can check that no non-identity element of $G$ fixes more than $\frac{1}{3}$ of the points. (Coincidentally, as far as we are aware, $\pi$ is the only permutation character of the Monster that has been computed.) For the rest of the proof, we may assume that $G_{\omega}$ is not conjugate to the double cover of the Baby monster. From [30, Section 3.6], it follows that $\left|G_{\omega}\right| \leq\left|2^{1+24} \cdot \mathrm{Co}_{1}\right|$. Now, it is an easy computation to check that

$$
\operatorname{fpr}_{\Omega}(g) \leq \frac{\left|g^{G} \cap G_{\omega}\right|}{\left|g^{G}\right|} \leq \frac{\left|G_{\omega}\right|}{\left|g^{G}\right|} \leq \frac{\left|2^{1+24} \cdot \mathrm{Co}_{1}\right|}{\left|g^{G}\right|}<\frac{1}{3},
$$

except when $g$ is in the conjugacy class $1 A$ and $2 A$. Therefore, for the rest of the proof, we may assume that $g$ is in the conjugacy class $2 A$. From [21, Lemma 2.7], we have

$$
\operatorname{fpr}_{\Omega}(g) \leq \max \left\{\frac{1+|\chi(g)|}{1+\chi(1)} \mid \chi \in \operatorname{Irr}(G), \chi(1) \neq 1\right\} .
$$

This quantity can be easily computed, and it is less than $\frac{1}{3}$.

This allows us to finish the proof of Theorem 1.1 when $T$ is a sporadic simple group. Let $\Sigma$ be a maximal system of imprimitivity for $G$ acting on $\Omega$. Except when $G=\operatorname{Aut}\left(M_{22}\right)$, the proof follows applying Lemmas 2.1 and 2.3 to $\Sigma$. When $G=\operatorname{Aut}\left(M_{22}\right)$, the proof follows directly from Lemma 2.3.

\subsection{Exceptional groups of Lie type}

Suppose now that the socle $T$ of $G$ is an exceptional group of Lie type. Exactly as for the sporadic groups, we prove a much stronger statement than needed for the proof of Theorem 1.1.

Lemma 2.4. Let $G$ be an almost simple primitive group on $\Omega$ with socle an exceptional simple group of Lie type. Then $\operatorname{fp}_{\Omega}(g) \leq \frac{1}{3}$ for every $g \in G \backslash\{1\}$.

Proof. For exceptional groups of Lie type, Lawther, Liebeck and Seitz [20, Theorem 1] have obtained useful and explicit upper bounds on $\operatorname{fpr}_{\Omega}(g)$. From these bounds, it readily follows that $\operatorname{fpr}_{\Omega}(g) \leq \frac{1}{3}$ for every element $g \in G \backslash\{1\}$, except when the socle of $G$ is $G_{2}(2)^{\prime}$ and ${ }^{2} G_{2}(3)^{\prime}$. Now, $G_{2}(2)^{\prime} \cong \operatorname{PSU}_{3}(3)$ and ${ }^{2} G_{2}(3)^{\prime} \cong \mathrm{PSL}_{2}(8)$; these cases can be analyzed with a help of a computer. The maximum fixed-point ratio for $G$ when the socle is $\mathrm{PSL}_{2}(8)$ is $\frac{1}{3}$ (arising from the natural action of $\mathrm{P \Gamma L}_{2}(8)$ on the nine points of the projective line). The same holds for groups having socle $\mathrm{PSU}_{3}(3)$; the maximum $\frac{1}{3}$ is achieved on the primitive action of degree 36 . 
The proof of Theorem 1.1 in the case when $T$ is an exceptional simple group of Lie type now follows by applying Lemmas 2.1 and 2.4 to a maximal system of imprimitivity $\Sigma$ of the action of $G$ on $\Omega$.

\subsection{Alternating groups}

Suppose now that $T$ (as an abstract group) is isomorphic to the alternating group $\operatorname{Alt}(n)$ for some $n \in \mathbb{N}$ with $n \geq 5$. For this proof, we argue by induction on $n$. We first consider the case that $n \in\{5,6,7,8\}$; this will avoid some detour in our arguments. The result in this case follows with a computation with the invaluable computer algebra system Magma [3]. From now on, we may assume that $n \geq 9$ and hence, in particular, $G=\operatorname{Alt}(n)$ or $G=\operatorname{Sym}(n)$. Since the action of $\operatorname{Alt}(n)$ on the cosets of one of its Sylow 2-subgroups extends to an action of $\operatorname{Sym}(n)$, we may assume that $G=\operatorname{Sym}(n)$.

Case " $n$ odd". Let $H$ be a subgroup of $G$ with $H \cong \operatorname{Sym}(n-1)$ and $G_{\omega} \leq H$ for some $\omega \in \Omega$. (Observe that this is possible because $n$ is odd.) Let $\Sigma$ be the system of imprimitivity determined by the overgroup $H$ of $G_{\omega}$. Clearly,

$$
\operatorname{Fix}_{\Omega}(g)=\bigcup_{B \in \operatorname{Fix}_{\Sigma}(g)} \operatorname{Fix}_{B}(g) .
$$

As $n-1 \geq 5$ and as $H$ acts faithfully on each of its orbits on $\Omega$, we deduce by induction that $\left|\operatorname{Fix}_{B}(g)\right| \leq \frac{1}{3}|B|$ for each $B \in \operatorname{Fix}_{\Sigma}(g)$. Therefore, $\operatorname{fpr}_{\Omega}(g) \leq \frac{1}{3}$. Case " $n$ even". Let $H$ be a subgroup of $G$ isomorphic to the imprimitive wreath product $\operatorname{Sym}\left(\frac{n}{2}\right)$ wr $\operatorname{Sym}(2)$ and with $G_{\omega} \leq H$ for some $\omega \in \Omega$. (Observe that this is possible because $n$ is even.) As above,

$$
\operatorname{Fix}_{\Omega}(g)=\bigcup_{B \in \operatorname{Fix}_{\Sigma}(g)} \operatorname{Fix}_{B}(g) .
$$

Let $B \in \operatorname{Fix}_{\Sigma}(g)$, and when $\operatorname{Fix}_{B}(g) \neq \emptyset$, let $\omega^{\prime} \in \operatorname{Fix}_{B}(g)$. Now, $G_{\omega} \leq G_{B} \cong H$, and hence, without loss of generality, we may suppose that $\omega^{\prime}=\omega$ and $G_{B}=H$. In what follows, we aim to estimate $\operatorname{Fix}_{B}(g)$.

Now, $G_{\omega}=P$ wr $\operatorname{Sym}(2)$, where $P$ is a Sylow 2-subgroup of $\operatorname{Sym}\left(\frac{n}{2}\right)$. The action of $H=\operatorname{Sym}\left(\frac{n}{2}\right)$ wr $\operatorname{Sym}(2)$ on the cosets of $P$ wr $\operatorname{Sym}(2)=G_{\omega}$ (that is, the action of $G$ on $\Omega$ ) is permutation equivalent to the natural product action of $\operatorname{Sym}\left(\frac{n}{2}\right)$ wr $\operatorname{Sym}(2)$ on the Cartesian product $\Delta^{2}$, where $\Delta=P \backslash \operatorname{Sym}\left(\frac{n}{2}\right)$. (This is clear from the structure of $H$ and $G_{\omega}$, and we refer to [29] for more details on Cartesian decompositions of permutation groups.) Now, we can write the element $g$ in the form $\left(h_{1}, h_{2}\right)$ or in the form $\left(h_{1}, h_{2}\right)(12)$, where $h_{1}, h_{2} \in P$ and (12) is the element swapping the two factors of $\Delta^{2}$, that is, $\left(\delta_{1}, \delta_{2}\right)^{(12)}=\left(\delta_{2}, \delta_{1}\right)$ for 
every $\left(\delta_{1}, \delta_{2}\right) \in \Delta$. A permutation of the second kind fixes at most $|\Delta|$ points, and hence

$$
\operatorname{fpr}_{\Omega}(g)=\operatorname{fpr}_{\Delta^{2}}(g) \leq \frac{1}{|\Delta|}=\frac{1}{\left|\operatorname{Sym}\left(\frac{n}{2}\right): P\right|} \leq \frac{1}{3} .
$$

A permutation of the first kind has the property that either $h_{1} \neq 1$ or $h_{2} \neq 1$. Since $\frac{n}{2} \geq 5$, we may apply induction and say that the non-identity element $h_{1}$ or $h_{2}$ fixes at most $\frac{1}{3}$ of its domain and hence so does $g$.

\subsection{Classical groups}

Suppose finally that the socle $T$ of $G$ is a simple classical group defined over the finite field of size $q$, and recall that we may assume that $T \leq G \leq \operatorname{Aut}(T)$.

Notation 2.5. For twisted groups, our notation for $q$ is such that $\operatorname{PSU}_{n}(q)$ and $\mathrm{P} \Omega_{2 m}^{-}(q)$ are the twisted groups contained in $\operatorname{PSL}_{n}\left(q^{2}\right)$ and $\mathrm{P}_{2 m}^{+}\left(q^{2}\right)$, respectively. We write $q=p^{e}$ for some prime $p$ and some $e \geq 1$, and we define

$$
q_{0}:= \begin{cases}q^{2} & \text { if } G \text { is unitary } \\ q & \text { otherwise. }\end{cases}
$$

We let $V$ be the natural module defined over the field $\mathbb{F}_{q_{0}}$ of size $q_{0}$ for the covering group of $T$, and we let $n$ be the dimension of $V$ over $\mathbb{F}_{q_{0}}$.

In studying actions of classical groups, it is rather natural to distinguish between those actions which permute the subspaces of the natural module and those which do not. The stabilizers of subspaces are generally rather large (every parabolic subgroup falls into this class), and therefore the fixed-point ratio in these cases also tends to be rather large. As the culmination of an important series of papers [5-8], Burness obtained remarkably good upper bounds on the fixed-point ratio for each finite almost simple classical group in non-subspace actions. For future reference, we first need to make the definition of non-subspace action precise.

Definition 2.6. Assume Notation 2.5. A subgroup $H$ of $G$ is a subspace subgroup if, for each maximal subgroup $M$ of $T$ that contains $H \cap T$, one of the following conditions holds:

(a) $M$ is the stabilizer in $T$ of a proper non-zero subspace $U$ of $V$, where $U$ is totally singular, or non-degenerate, or, if $T$ is orthogonal and $p=2$, a nonsingular 1-subspace ( $U$ can be any subspace if $T=\operatorname{PSL}(V)$ );

(b) $M=\mathrm{O}_{2 m}^{ \pm}(q)$ and $(T, p)=\left(\operatorname{Sp}_{2 m}(q)^{\prime}, 2\right)$. 
A transitive action of $G$ on a set $\Delta$ is a subspace action if the point stabilizer $G_{\delta}$ of $\delta \in \Delta$ is a subspace subgroup of $G$; non-subspace subgroups and actions are defined accordingly. For the convenience of the reader, we recall [5, Theorem 1].

Theorem 2.7 ([5, Theorem 1]). Let $G$ be a finite almost simple classical group acting transitively and faithfully on a set $\Delta$ with point stabilizer $G_{\delta} \leq H$, where $H$ is a maximal non-subspace subgroup of $G$. Let $T$ be the socle of $G$. Then, for every $x \in G \backslash\{1\}$, we have $\operatorname{fpr}_{\Omega}(x)<\left|x^{G}\right|^{-\frac{1}{2}+\frac{1}{n}+\iota}$, where $n$ is defined in $[5$, Definition 2] and either $\iota=0$ or $(T, H, \iota)$ is listed in [5, Table 1].

In Theorem 2.7, apart from $\mathrm{PSL}_{3}(2)$ and $\mathrm{PSp}_{4}(2)^{\prime}$ (where $n=2$ ), $n$ is exactly as in Notation 2.5. The upper bound in Theorem 2.7 is quite sharp when $n$ is large and is extremely useful for our application. However, for small values of $n$, Theorem 2.7 loses all of its power. For instance, when $T=\mathrm{PSL}_{4}(q)$, we see from [5, Table 1] that $\iota=\frac{1}{4}$, and hence the upper bound in Theorem 2.7 only says $\operatorname{fpr}_{\Omega}(x) \leq\left|x^{G}\right|^{0}=1$. However, we point out that Burness and Guest [9] have strengthened Theorem 2.7 for linear groups of very small rank.

We are now well equipped for the proof of Theorem 1.1 in the case where $T$ is a non-abelian simple classical group. We use the notation that we have established above. We argue by contradiction, and we suppose that there exists $g \in G \backslash\{1\}$ with $\operatorname{fpr}_{\Omega}(g)>\frac{1}{3}$. Without loss of generality, we may assume that $g$ is an involution. Recall that we are proving Theorem 1.1 by induction on $|\Omega|+|G|$. In particular, we may suppose that $G=\langle T, g\rangle$.

Case " $q \geq 4$ ". When $q \geq 4,\left[21\right.$, Theorem $\left.1^{\prime}\right]$ yields that the pair $(T, g)$ is in $[21$, Tables 1 and 2]. These tables, together with the pair $(T, g)$, have some additional information on the subgroup $G_{\omega}$, on a maximal subgroup $M$ of $G$ containing $G_{\omega}$ and on $\operatorname{fpr}_{\Omega}(g)$. Using this detailed information, a routine case-by-case analysis yields that $\operatorname{fpr}_{\Omega}(x) \leq \frac{1}{3}$ for every $x \in G \backslash\{1\}$.

Case " $q \leq 3$ and $n \leq 8$ ". Since we have only a finite number of cases to check, we have proved the result invoking the help of a computer. This was done with a very naive algorithm, based on Lemma 2.1, which we now briefly explain. Given a group $X$ and a collection of subgroup $y$ of $X$, we construct all maximal subgroups $\mathcal{M}_{Y}$ of $Y$ for each $Y \in \mathcal{Y}$. For each $M \in \mathcal{M}_{Y}$, we construct the permutation representation of $X$ acting on $M \backslash X$. If $X$ contains a non-identity permutation with $\operatorname{fpr}_{M \backslash X}(x)>\frac{1}{3}$, then we save $M$ in a set $Y^{\prime}$; otherwise, we disregard $M$ from further analysis. Next, we apply this routine with $y$ replaced by $y^{\prime}$. (This computation might seem very time and memory consuming, but for most groups, this procedure stops after the first iteration.) Finally, we consider the collection $\mathcal{X}$ of all subgroups returned in the previous procedure and check that the claim of the theorem holds in each case. 
For the rest of the proof, we may assume $q \leq 3$ and $n \geq 9$.

Case " $G_{\omega} \leq H$, where $H$ is a maximal non-subspace subgroup of $G$ ". We use the result of Burness described earlier. Then $\frac{1}{3}<\left|\operatorname{fpr}_{\Omega}(g)\right|<\left|g^{G}\right|^{-\frac{1}{2}+\frac{1}{n}+\iota}$ and

$$
\left|g^{G}\right| \leq 3^{\frac{1}{2}-\frac{1}{n}-\iota} .
$$

Using the information on $\iota$ in [5, Table 1], some very basic information on the conjugacy classes of $T$ (which can be find in [14] or in [12] for small groups) and $n \geq 9$, we found with a case-by-case analysis that (2.3) is never satisfied.

For the rest of the proof, we may assume that the only maximal subgroups $H$ of $G$ with $G_{\omega} \leq H$ are subspace subgroups. Definition 2.6 (b) does not arise here because $G_{\omega}$ is a Sylow 2-subgroup of $G$ (recall (2.1)), but $\left|\operatorname{Sp}_{2 m}(q): \mathrm{O}_{2 m}^{ \pm}(q)\right|$ is even. In particular, every maximal subgroup $H$ of $G$ with $G_{\omega} \leq H$ is in the Aschbacher class $\mathscr{C}_{1}$.

Case "there exists a maximal subgroup $H$ of $G$ with $G_{\omega} \leq H, \mathbf{O}_{2}(H) \cap g^{G}=\emptyset$ ". Let $\Sigma$ be the system of imprimitivity determined by the overgroup $H$ of $G_{\omega}$. Consider $\Delta:=\omega^{H}$ and the permutation group $H^{\Delta}$ induced by $H$ on $\Delta$. Since $G_{\omega}$ is a Sylow 2-subgroup of $G$, we deduce that $G_{\omega}$ is a Sylow 2-subgroup of $H$. Therefore, the kernel of the action of $H$ on $\Delta$ is

$$
\bigcap_{h \in H} G_{\omega}^{h}=\mathbf{O}_{2}(H)
$$

Thus $H^{\Delta} \cong H / \mathbf{O}_{2}(H)$, and hence $\mathrm{O}_{2}\left(H^{\Delta}\right)=1$. We have

$$
\operatorname{Fix}_{\Omega}(g)=\bigcup_{\Delta^{\prime} \in \operatorname{Fix}_{\Sigma}(g)} \operatorname{Fix}_{\Delta^{\prime}}(g) .
$$

Let $\Delta^{\prime} \in \operatorname{Fix}_{\Sigma}(g)$. Then $\Delta^{\prime}=\Delta^{x}$ for some $x \in G$, and $H^{x}$ is the setwise stabilizer of $\Delta^{\prime}$ in $G$. Therefore, $\mathbf{O}_{2}\left(\left(H^{x}\right)^{\Delta^{\prime}}\right)=1$. Since $\left|\Delta^{\prime}\right|<|\Omega|$, by induction, we have that either $\operatorname{fpr}_{\Delta^{\prime}}(g) \leq \frac{1}{3}$ or $g$ fixes pointwise each element of $\Delta^{\prime}$. However, the latter case does not arise because the kernel of the action of $H^{x}$ on $\Delta^{\prime}$ is $\mathbf{O}_{2}(H)^{x}$, and we are assuming $\mathbf{O}_{2}(H) \cap g^{G}=\emptyset$. Thus $g$ does not act trivially on $\Delta^{\prime}$ and $\operatorname{fpr}_{\Delta^{\prime}}(g) \leq \frac{1}{3}$. Now, (2.4) yields $\operatorname{fpr}_{\Omega}(g) \leq \frac{1}{3}$.

For the rest of the proof, we may suppose that every maximal subgroup $H$ of $G$ with $G_{\omega} \leq H$ is in the Aschbacher class $\ell_{1}$ and satisfies $\mathbf{O}_{2}(H) \cap g^{G} \neq \emptyset$. In particular, $\mathbf{O}_{2}(H) \neq 1$, and hence $H$ is a 2-local subgroup. Using the information on the maximal subgroups $H$ of the finite classical groups in the Aschbacher class $\zeta_{1}$ in [19, Section 4.1] and the fact that $\mathbf{O}_{2}(H) \cap g^{G} \neq \emptyset$, we obtain $g \in T$ and $G=\langle T, g\rangle=T$. 
Case " $q=3$ ". Using again the information in [19, Section 4.1], we have that if $H$ is a maximal subspace subgroup with $G_{\omega} \leq H$ and $q=3$, then $\left|\mathbf{O}_{2}(H)\right| \leq 2$. In particular, we are in the position to refine slightly the argument in (2.4). Let $\Sigma$ be the system of imprimitivity determined by the overgroup $H$ of $G_{\omega}$. We partition Fix $_{\Sigma}(g):=\Sigma_{1} \cup \Sigma_{2}$ in two subsets: $\Sigma_{1}$ consists of the $\Delta \in$ Fix $_{\Sigma}(g)$ with $g$ not fixing pointwise $\Delta$, and $\Sigma_{2}$ consists of the $\Delta \in \operatorname{Fix}_{\Sigma}(g)$ with $g$ fixing pointwise $\Delta$. Observe that if $\Delta \in \Sigma_{2}$, then $g \in \mathbf{O}_{2}\left(G_{\{\Delta\}}\right)$, and hence $\mathbf{O}_{2}\left(G_{\{\Delta\}}\right)=\langle g\rangle$. If $\Sigma_{2}$ contains two distinct elements $\Delta$ and $\Delta^{\prime}$, then $\mathbf{O}_{2}\left(G_{\{\Delta\}}\right)=\langle g\rangle=\mathbf{O}_{2}\left(G_{\left\{\Delta^{\prime}\right\}}\right)$, and hence $\langle g\rangle$ is centralized by $\left\langle G_{\{\Delta\}}, G_{\left\{\Delta^{\prime}\right\}}\right\rangle=G$, where the last equality follows from the maximality of $G_{\{\Delta\}}$ and $G_{\left\{\Delta^{\prime}\right\}}$ in $G$ and from $\Delta \neq \Delta^{\prime}$. Therefore, $\left|\Sigma_{2}\right| \leq 1$. Thus, applying the inductive hypothesis for the action of $g$ on $\Delta \in \operatorname{Fix}_{\Sigma}(g)$, from (2.4), we deduce

$$
\begin{aligned}
\left|\operatorname{Fix}_{\Omega}(g)\right| & =\sum_{\Delta \in \Sigma_{1}}\left|\operatorname{Fix}_{\Delta}(g)\right|+\sum_{\Delta \in \Sigma_{2}}\left|\operatorname{Fix}_{\Delta}(g)\right| \\
& \leq\left|\Sigma_{1}\right| \frac{\left|\omega^{H}\right|}{3}+\left|\Sigma_{2}\right|\left|\omega^{H}\right| \leq\left|\omega^{H}\right|\left(\frac{\left|\operatorname{Fix}_{\Sigma}(g)\right|-1}{3}+1\right) .
\end{aligned}
$$

Since $g \neq 1, g$ does not act trivially on $\Sigma$, and hence $\left|\operatorname{Fix}_{\Sigma}(g)\right| \leq|\Sigma|-2$. Thus

$$
\left|\operatorname{Fix}_{\Omega}(g)\right| \leq\left|\omega^{H}\right|\left(\frac{|\Sigma|-3}{3}+1\right)=\frac{\left|\omega^{H}\right||\Sigma|}{3}=\frac{|\Omega|}{3} .
$$

Case " $q=2$ ”. Here $G=\langle T, g\rangle$ is one of the groups $\operatorname{PSL}_{n}(2), \operatorname{PSU}_{n}(2), \operatorname{PSp}_{n}(2)$, $\mathrm{P} \Omega_{n}^{+}(2)$ and $\mathrm{P} \Omega_{n}^{-}(2)$.

Recall that $V$ is the underlying module for $G$. Let $W$ be a totally isotropic subspace of $V$ of dimension 1 fixed by $G_{\omega}$, and let $H$ be the stabilizer of $W$. (The existence of $W$ is guaranteed by the fact that $G_{\omega}$ is a 2-group and by the fact that $V$ has characteristic 2.) Now, $H$ is a maximal $\ell_{1}$-subgroup of $G$ with $G_{\omega} \leq H$. Considering the cases we are left at this point of the proof, $H$ is a maximal parabolic subgroup of $G$. Since $\mathbf{O}_{2}(H) \cap g^{G} \neq \emptyset$, we deduce from the structure of $H$ in [19] that $g$ is a transvection, that is, $\operatorname{dim} \mathbf{C}_{V}(g)=n-1$.

Let $U$ be the group of upper unitriangular matrices in $\mathrm{GL}_{n}(2)$, and let $T(n)$ be the number of transvections in $U$. It is easy to show arguing inductively on $n$ that $T(n)=2 T(n-1)+2^{n-1}-1$. Using this recursive relation and the fact that $T(2)=1$, we obtain $T(n)=(n-2) 2^{n-1}+1$.

From the previous paragraph, $\left|g^{G} \cap G_{\omega}\right| \leq(n-2) 2^{n-1}+1$, and hence

$$
\operatorname{fpr}_{\Omega}(g)=\frac{\left|g^{G} \cap G_{\omega}\right|}{\left|g^{G}\right|} \leq \frac{(n-2) 2^{n-1}+1}{\left|G: \mathbf{C}_{G}(g)\right|} \leq \frac{1}{3},
$$


where the last inequality follows by comparing $\left|G: \mathbf{C}_{G}(g)\right|$ with $(n-2) 2^{n-1}+1$. (Information on $\left|G: \mathbf{C}_{G}(g)\right|$ can be found for all the groups under consideration either in [19] or in [14].)

This completes the proof of Theorem 1.1.

\section{Bibliography}

[1] R. Abbott, J. Bray, S. Linton, S. Nickerson, S. Norton, R. Parker, I. Suleiman, J. Tripp, P. Walsh and R. Wilson, AtlasRep - a GAP package, Version 1.5.1, 2016, https://www.gap-system.org/Packages/atlasrep.html.

[2] L. Babai, On the order of uniprimitive permutation groups, Ann. of Math. (2) 113 (1981), 553-568.

[3] W. Bosma, J. Cannon and C. Playoust, The Magma algebra system. I. The user language, J. Symbolic Comput. 24 (1997), 235-265.

[4] T. Breuer and K. Lux, The multiplicity-free permutation characters of the sporadic simple groups and their automorphism groups, Comm. Algebra 24 (1996), 22932316.

[5] T. Burness, Fixed point ratios in actions of finite classical groups I, J. Algebra 309 (2007), 69-79.

[6] T. Burness, Fixed point ratios in actions of finite classical groups II, J. Algebra 309 (2007), 80-138.

[7] T. Burness, Fixed point ratios in actions of finite classical groups III, J. Algebra 314 (2007), 693-748.

[8] T. Burness, Fixed point ratios in actions of finite classical groups IV, J. Algebra 314 (2007), 749-788.

[9] T. Burness and S. Guest, On the uniform spread of almost simple linear groups, Nagoya Math. J. 209 (2013), 37-110.

[10] M. Conder and P. Dobcsányi, Trivalent symmetric graphs on up to 768 vertices, J. Combin. Math. Combin. Comput. 40 (2002), 41-63.

[11] M. Conder, Trivalent (cubic) symmetric graphs, preprint (2020), https://www . math.auckland.ac.nz/ conder/symmcubic10000list.txt.

[12] J. H. Conway, R. T. Curtis, S. P. Norton, R. A. Parker and R. A. Wilson, Atlas of Finite Groups: Maximal Subgroups and Ordinary Characters for Simple Groups, Oxford University, Eynsham, 1985.

[13] D. Gluck and K. Magaard, Character and fixed point ratios in finite classical groups, Proc. Lond. Math. Soc. (3) 71 (1995), 547-584. 
[14] D. Gorenstein, R. Lyons and R. Solomon, The Classification of the Finite Simple Groups, Math. Surveys Monogr. 40, American Mathematical Society, Providence, 1998.

[15] S. Guest, J. Morris, C. E. Praeger and P. Spiga, On the maximum orders of elements of finite almost simple groups and primitive permutation groups, Trans. Amer. Math. Soc. 367 (2015), no. 11, 7665-7694.

[16] R. Guralnick and K. Magaard, On the minimal degree of a primitive permutation group, J. Algebra 207 (1998), 127-145.

[17] M. Isaacs, Character Theory of Finite Groups, Academic Press, New York, 1976.

[18] J. Kempe, L. Pyber and A. Shalev, Permutation groups, minimal degrees and quantum computing, Groups Geom. Dyn. 1 (2007), 553-584.

[19] P. Kleidman and M. Liebeck, The Subgroup Structure of the Finite Classical Groups, London Math. Soc. Lecture Note Ser. 129, Cambridge University, Cambridge, 1990.

[20] R. Lawther, M. W. Liebeck and G. M. Seitz, Fixed point ratios in actions of finite exceptional groups of Lie type, Pacific J. Math. 205 (2002), 393-464.

[21] M. Liebeck and J. Saxl, Minimal degrees of primitive permutation groups, with an application to monodromy groups of covers of Riemann surfuces, Proc. Lond. Math. Soc. (3) 63 (1991), 266-314.

[22] M. Muzychuk and P. Spiga, Finite primitive groups of small rank: Symmetric and sporadic groups, J. Algebraic Combin. 52 (2020), 103-136.

[23] M. F. Newman, E. A. O'Brien and A. Shalev, The fixity of groups of prime-power order, Bull. Lond. Math. Soc. 27 (1995), 225-231.

[24] P. Potočnik and P. Spiga, On the number of fixed points of automorphisms of vertextransitive graphs, preprint (2019) https://arxiv.org/abs/1909.05456; to appear in Combinatorica.

[25] P. Potočnik, P. Spiga and G. Verret, Cubic vertex-transitive graphs on up to 1280 vertices, J. Symbolic Comput. 50 (2013), 465-477.

[26] P. Potočnik, P. Spiga and G. Verret, A census of 4-valent half-arc-transitive graphs and arc-transitive digraphs of valence two, Ars Math. Contemp. 8 (2015), 133-148.

[27] C. E. Praeger, An O'Nan-Scott Theorem for finite quasiprimitive permutation groups and an application to 2-arc transitive graphs, J. Lond. Math. Soc. (2) 47 (1993), 227-239.

[28] C. E. Praeger, Finite quasiprimitive graphs, in: Surveys in Combinatorics, London Math. Soc. Lecture Note Ser. 24, Cambridge University, Cambridge (1997), 65-85.

[29] C. E. Praeger and C. Schneider, Permutation Groups and Cartesian Decompositions, London Math. Soc. Lecture Note Ser. 449, Cambridge University, Cambridge, 2018. 
[30] R. A. Wilson, Maximal subgroups of sporadic groups, in: Finite Simple Groups: Thirty Years of the Atlas and Beyond, Contemp. Math. 694, American Mathematical Society, Providence (2017), 57-72.

[31] M.-M. Zhang and J.-X. Zhou, Tetravalent half-arc-transitive bi- $p$-metacirculants, J. Graph Theory 92 (2019), 19-38.

[32] J.-X. Zhou, Tetravalent half-arc-transitive p-graphs, J. Algebraic Combin. 44 (2016), 947-971.

[33] The GAP Group, GAP - Groups, Algorithms, and Programming, Version 4.8.7, 2017, http://www. gap-system.org.

Received April 14, 2020; revised December 2, 2020.

\section{Author information}

Primož Potočnik, Faculty of Mathematics and Physics,

University of Ljubljana, Jadranska 21, SI-1000;

and Institute of Mathematics, Physics, and Mechanics,

Jadranska 19, SI-1000 Ljubljana, Slovenia.

E-mail: primoz.potocnik@fmf.uni-lj.si

Corresponding author:

Pablo Spiga, Dipartimento di Matematica e Applicazioni,

University of Milano-Bicocca, Via Cozzi 55, 20125 Milano, Italy.

E-mail: pablo.spiga@unimib.it 